\title{
Analysis of intergeneric sexual hybridization between transgenic Brassica oleracea and Sinapis alba
}

\author{
Jun Li $\cdot$ Chunlei Zhang $\cdot$ Chunyun Guan $\cdot$ Lixia Luo $\cdot$ Li Ren • \\ Wenhui Wei $\cdot$ Guangyuan Lu $(\mathbb{0} \cdot$ Xiaoping Fang
}

Received: 14 July 2017 / Accepted: 6 November 2017/Published online: 13 November 2017

(C) The Author(s) 2017. This article is an open access publication

\begin{abstract}
Sinapis alba possesses a number of desirable traits that can be employed to broaden genetic variability in Brassica oleracea or B. napus. Using transgenic $B$. oleracea $(\mathrm{CC}, 2 \mathrm{n}=18)$ as paternal plants and non-transgenic $S$. alba $(\mathrm{SS}, 2 \mathrm{n}=24)$ as maternal plants, 11 hybrid plants were successfully obtained by using a combination of ovary culture and embryo rescue, while 2 seedlings of the reciprocal cross were generated but lost during the propagation. The hybridity of these plants was confirmed by flower color and other agronomic characteristics, chromosome counting, and pollen viability. Most of the
\end{abstract}

J. Li · W. Wei · G. Lu (

Key Laboratory of Biology and Genetic Improvement of Oil Crops of the Ministry of Agriculture, Oil Crops

Research Institute, Chinese Academy of Agricultural Sciences, Wuhan, China

e-mail: luwiz@163.com

J. Li · C. Zhang $\cdot$ L. Luo $\cdot$ L. Ren $\cdot$ X. Fang $(\bowtie)$

Key Laboratory of Crop Cultivation and Physiology of the Ministry of Agriculture, Oil Crops Research Institute, Chinese Academy of Agricultural Sciences, Wuhan, China

e-mail: fangxp@oilcrops.cn

C. Guan

College of Agriculture, Hunan Agricultural University, Changsha, China

G. $\mathrm{Lu}$

New Rural Development Institute, Hubei Engineering University, Xiaogan, China intergeneric $F_{1}$ plants showed only one set each of $\mathrm{S}$ and $\mathrm{C}$ chromosome $(\mathrm{SC}, 2 \mathrm{n}=21)$ and were male sterile, while a few plants were semi-fertile and had one set of $\mathrm{S}$ chromosomes but two sets of $\mathrm{C}$ chromosomes (CCS, 2n = 30). PCR, genomic Southern blotting and qRT-PCR for bar gene revealed that among the $\mathrm{F}_{1}$ hybrid plants only $9.1 \%$ was bar positive and could be forwarded to $F_{2}$ and $F_{3}$ generation. The majority of $F_{3}$ plants obtained sufficient resistance to Alternaria brassicae. Thus, the generation of disease (A. brassicae) and herbicide (Bastar) resistant intergeneric hybrids was of importance for breeding program. The existence of bar gene in these intermediate materials will facilitate the identification of hybridity and transfer of S.alba traits into target genetic background.

Keywords Brassica oleracea - Sinapis alba . Intergeneric hybridization $\cdot$ Bar gene $\cdot$ Disease resistance $\cdot$ Alternaria brassicae

\section{Introduction}

Approximately 330 genera and 3700 species are included in the Brassicaceae family. Among these, the genus Brassica contains many economically important crops for oil, feed, condiment and vegetable such as B. oleracea $(\mathrm{CC}, 2 \mathrm{n}=18)$ (Kaneko and 
Bang 2014). The B. oleracea is one of the most valuable crops and has provided humans with healthy vegetable for hundreds of years. B. oleracea comprises multiple, distinct types and is widely cultivated in China. However, $B$. oleracea has weak tolerance to frost, mildew, aphids, black spot, and black rot diseases and is also susceptible to abiotic stresses such as drought (Gribova et al. 2006; Wei et al. 2007), which may cause severe yield loses during production. Therefore, introduction of desired traits into $B$. oleracea from other related species is of great interest.

Sinapis alba $(\mathrm{SS}, 2 \mathrm{n}=24)$ is a species within Brassicaceae that is phylogenetically close to Brassica species and has many desirable agronomic traits, including tolerance to high temperatures and drought stress and resistance to a variety of diseases such as black spot (Hansen and Earle 1997), flea beetles (Bodnaryk and Lamb 1991) and clubroot (Lelivelt et al. 1993). However, S. alba has historically been developed only for condiment use because of its poor oil quality and high level of glucosinolates in the seed (Brown et al. 1997). Nevertheless, it is an attractive genetic stock for interspecific or intergeneric hybridization for germplasm enhancement, and a new variety with double low quality was recently developed in Poland (Pietka et al. 2014).

The fungal pathogen Alternaria brassicae can cause severe blackspot disease in the Brassicaceae, including three of the most important vegetable crops in the world, namely cauliflower, broccoli and cabbage. Moreover, if the disease continues to be aggravated on any part of a plant with spotting symptoms, the market value and production of vegetables will be greatly reduced. However, efforts to idenfify sources of high resistance to A. brassicae among related species that could be readily crossed with B. oleracea have failed (Hansen and Earle 1997).

Introduction of economically important traits from their wide relatives into Brassica crops via interspecific or intergeneric hybridization is recognized as a promising strategy. Successful hybridizations between S. alba and B. napus have been achieved by sexual or somatic hybridization (Lelivelt et al. 1993; Wang et al. 2005). Similarly, the crossability between $S$. alba and B. rapa has been confirmed (Gong et al. 1994). Likewise, Hansen and Earle (1997) carried out the somatic hybridization between $S$. alba and B. oleracea by protoplast fusion. However, somatic hybridization may generate chimeric lines and the plants obtained always showed very poor pollen fertility (Chevre et al. 1994; Nothnagel et al. 1997; Chen et al. 2005; Wang et al. 2011). To avoid such shortcomings, we prefer to use sexual hybridization and embryo rescue techniques to produce intergeneric hybrid lines of $S$. alba and $B$. oleracea, In this study, we aimed to illustrate the sexual crossability between the transgenic $B$. oleracea and non-transgenic $S$. alba and further assess the potential utilization of such hybrids as intermediate genetic stock in breeding.

\section{Materials and Methods}

Plant materials

Transgenic cv. 'Hong-Kong Zhonghua Jielan' (B. oleracea var. alboglabra, genome $\mathrm{CC}, 2 \mathrm{n}=18$ ) and non-transgenic accession 'Xinjiang Baijie' (Sinapis alba L., white mustard var. Asta, genome SS, $2 \mathrm{n}=24$ ) plants were used in the intergeneric hybridization. Previously, the herbicide resistant bar gene was transferred into the genome of $B$. oleracea via agrobacterium mediated transformation ( $\mathrm{Li}$ et al. 2006). A homozygous $T_{2}$ line (term ' $B$. oleracea-bar' hereafter) was chosen for this study. Seeds of the two lines along with their offspring were grown in pots in glass house or in field of Hanchuan Transgenic Experimental Station attached to Oil Crops Research Institute, CAAS (certificated by Chinese Government).

\section{Intergeneric hybridizations}

Reciprocal intergeneric hybridizations between $S$. $a l b a$ and bar transgenic B. oleracea plants were conducted. The fully developed young buds were hand emasculated one day before flowering, and immediately pollinated with fresh pollens and topped with a paper bag to prevent cross pollen contamination. In total $\sim 1200$ crosses were made. Seven days after pollination, ovaries (young fruits) were excised from plants, surface sterilized (70\% alcohol for 5-10 s, 1\% DICA for 8-10 min, washed with sterile water 3-4 times) and cultured on MS medium supplemented with $500 \mathrm{mg} / \mathrm{L}$ hydrolyzed casein (with $0.8 \%$ agar and $3.0 \%$ sucrose) for approximately 35-40 days. Then, ovules, if any, were dissected from the fruits and 
inoculated into MS medium until they grew into normal seedlings. Such seedlings were then cut off and treated in MS medium with $0.01 \%$ colchicine for 7-10 days for chromosome doubling and transferred into the rooting medium (MS $+0.2 \mathrm{mg} / \mathrm{L} \mathrm{NAA}$ ) for root initiation and multiplication. After robust roots developed, the plants were transplanted into pots and grown with appropriate shade and moisture.

\section{Cytological test}

The chromosome numbers of the hybrids was determined using young buds. Pistils of flower buds were peeled out, treated with $0.002 \mathrm{~mol} / \mathrm{L}$ 8-hydroxyquinoline for $4 \mathrm{~h}$, then fixed in Carnoy's solution for $24 \mathrm{~h}$ and stored in $70 \%$ ethanol at $4{ }^{\circ} \mathrm{C}$ until use. The pistils were hydrolyzed in $1.0 \mathrm{~mol} / \mathrm{L} \mathrm{HCl}$ at $60{ }^{\circ} \mathrm{C}$ for approximately $10 \mathrm{~min}$, squashed in a drop of modified carbolfuchsin and observed under a microscope.

Genomic in situ hybridization (GISH) assays were conducted according to the protocol described by Wei et al. (2007), with minor modifications. Briefly, chromosome preparations were treated with $80 \mu \mathrm{g} /$ $\mathrm{mL}$ RNase at $37{ }^{\circ} \mathrm{C}$ for $1 \mathrm{~h}$ and then rinsed gently in $2 \times$ SSC. Chromosomal DNA was denatured by immersing the slide in $70 \%$ deionized formamide at $70{ }^{\circ} \mathrm{C}$ for $2 \mathrm{~min}$. After dehydration of the preparation in an ice-cold 70, 95 and $100 \%$ ethanol series and air drying, $40 \mu \mathrm{L}$ of denatured probe cocktail was placed on a slide and hybridization was performed at $37{ }^{\circ} \mathrm{C}$ for $12 \mathrm{~h}$. Post-hybridization washes to remove weakly bound probe included a stringent wash in $20 \%$ formamide, a wash in $2 \times$ SSC and a wash in $0.1 \times \mathrm{SSC}$ at $42^{\circ} \mathrm{C}$ for $10 \mathrm{~min}$ to remove weakly bound probe. For doublecolor GISH, signals were first detected with Streptavidin-Cy3 (GE Healthcare Bio-Sciences, Pittsburgh, USA, Cat. No. PA43001) and then washed in PBS buffer for $10 \mathrm{~min}$, followed by immediate sequential detection with Anti-Digoxigenin-Fluorescein (Roche, Penzberg, Bayern, Germany, Cat. No. 1207741) and another wash in PBS buffer for $10 \mathrm{~min}$. For single-color GISH, only Streptavidin$\mathrm{Cy} 3$ or Anti-Digoxigenin-Fluorescein was used. Slides were counterstained with $2 \mu \mathrm{g} / \mathrm{mL}$ DAPI (4,6-diamidino-2-phenylindole) and examined under a Leica DM IRB fluorescence microscope assembled with DFC300 CCD and FW4000 software.
Pollen viability

To determine the pollen fertility, anthers from justopening flowers were dissected and squashed in $1 \%$ acetocarmine and examined under a microscope. The pollen viability rate was calculated as the proportion of stained pollen grains in total pollen grains scored $\times 100$. Three fields of view per flower were examined for a total of approximately 800 pollen grains.

Seed weight and oil content assessment

The Marvin analyzer (GTA Sensorik GmbH, Neubrandenburg, Germany) was used for seed weight measurement. Around $0.3 \mathrm{~g}$ seeds were first weight by the electronic balance connected to the computer and then spread across the sample tray for a snapshot, from which the number of seeds and 1000-seed-weight were automatically calculated.

To measure the oil content of seeds in small seed quantity, we employed a method developed by Wei et al. (2008) rather than using the common Near Infrared Reflectance Spectroscopy method that required a large number of seeds. In brief, $\sim 20 \mathrm{mg}$ seeds were quantified and then crushed to the particle size $<0.5 \mathrm{~mm}$ and oils were extracted by petroleum ether for $60 \mathrm{~min}$ in ultrasound cleaning bath KQ500DB (Ultrasound instrument Co., Ltd, Kunshan, China). KOH-methanol solution was added to the extract for the fatty acid methyl esters preparation. After centrifugation for $10 \mathrm{~min}$ at $4500 \mathrm{rpm}$, the supernatant of the solution was taken for gas chromatography analysis.

\section{Herbicide resistance test and molecular detection}

To detect herbicide resistance in field, the herbicide $\left(13.5 \%\right.$ Basta $^{\circledR}$ at a 1:200 dilution) was sprayed on the leaves of hybrid lines, after 5 days the syndrome of phytotoxicity on the leaves were investigated. To confirm the resistance of herbicide in the lab, seeds were sown in culture medium amended with $15 \mathrm{mg} / \mathrm{L}$ L-Phosphinothricin (PPT, active ingredient of Basta $^{\circledR}$ ) and the growth status of plants was recorded after 10 days. Non-transgenic B. oleracea plants were used as negative controls.

Southern blotting analysis was conducted as follows: $25 \mu \mathrm{g}$ of total genomic DNA was digested 
completely with EcoR I and electrophoretically separated on $0.8 \%$ agarose gel. For probe labeling and detection, a digoxin labeling kit was used (Roche Diagnostics, Swiss). A PCR-generated 0.5-kb fragment of the bar gene was used as the template for probe labeling.

Real-time quantitative PCR was performed on a Roche LightCycler480 system by using the SYBR green PCR kit (TOYOBO, Japan). Based on previous research, gene CruA (Accession No. X14555) was selected as the endogenous reference according to previous research (Wu et al. 2010). qPCR was performed according to the following protocol: $10 \mathrm{~s}$ denaturation at $95{ }^{\circ} \mathrm{C}, 15 \mathrm{~s}$ annealing at $56^{\circ} \mathrm{C}$, and 20 s elongation at $72{ }^{\circ} \mathrm{C}$ in 42 cycles. Specific primers for the bar gene were the following: qbarF: 5'-ACAAGCACGGTCAACTTCC-3'; qbarR: 5'-ACT CGGCCGTCCAGTTGGTA-3' . Data were analyzed in triplicate using independent cDNA samples.

\section{Assessment of resistance to Alternaria brassicae}

Thirty-eight 4-week-old hybrid lines and ten each of the parents were analyzed for resistance to A. brassicae according to a previously described protocol, with minor modifications (Scholze et al. 2010). Briefly, A. brassicae was cultivated on the vegetable juice medium at $20{ }^{\circ} \mathrm{C}$ without light. The conidia were then suspended in sterile water and adjusted to approximately $7 \times 10^{4}$ spores $/ \mathrm{mL}$. Plants were inoculated with the conidia suspension and then incubated for approximately 6 days. The disease severity was assessed on a scale of $0-8$, which was modified according to Hansen and Earle (1997) with 0 showing no disease symptoms, 1-2 indicating high resistance, 3-4 moderate resistance, 5-7 increasing susceptibility and 8 was a completely dead plant. The tests were repeated three times.

\section{Results}

Intergeneric sexual hybridization

between transgenic B. oleracea and S. alba

In the glass house, the maternal non-transgenic $S$. alba plants were pollinated by the transgenic $B$. oleracea plants. A total of 410 ovaries were obtained, which were then further grew on MS medium. After cultivation in vitro for 40 days, 17 embryos were rescued, which later grew into 13 putative intergeneric hybrid seedlings (Fig. 1). The estimated success rate was very low (3.17\%, Table 1$)$, indicating that it is difficult but possible to develop intergeneric hybrid between $S$. alba and B. oleracea by sexual hybridization. The seedlings were transplanted into the field, where 11 out of $13 F_{1}$ plants survived (JF2601-2611, Table 2).

The Reciprocal crosses (i.e. B. oleracea-bar $\times S$. alba) were also made, which yielded only 152 ovaries, mainly due to a lower fertilization frequency. Similarly, the post-pollinated ovaries were cultured on MS media. This procedure generated only 6 embryos and finally 2 hybrid seedlings. The percentage of seedling obtained was extremely low $(1.32 \%$, Table 1$)$, meaning that it is even more difficult to generate intergeneric hybrid by using $B$. oleracea as female parent. Unfortunately, these two $F_{1}$ hybrid seedlings were both vitrified during media cultivation and could not grew into mature plants for further analysis.

Moreover, approximately 600 sexual crosses (both direct and reciprocal) were also performed under field conditions (without embryo rescue) in the Transgenic Experimental Station, but no seed setting was observed in any of the crosses, indicating that natural occurrence of intergeneric hybrid is unlikely to happen between B. oleracea-bar and S. alba, at least for the accessions tested in this study.

Transgene detection

To determine whether the bar gene has been incorporated into the intergeneric hybrid, PCR assays were performed using genomic DNAs from each of the 11 $\mathrm{F}_{1}$ hybrid plants obtained. Compared with the result for the positive control, only one bar positive line (JF2608) was detected that had the same single DNA band (Fig. 2a), indicating that the frequency of intergeneric hybrid with bar gene was $9.1 \%$. The results of Southern blot analysis indicated that only one copy of the exogenous bar gene inserted into the male parent $B$. oleracea genome and confirmed that the bar gene had been integrated into the genome of hybrid line JF2608 (Fig. 2b). Real-time quantitative PCR assay was further performed to quantified the expression level of bar gene, which revealed that it was highly expressed only in samples of the male parent $B$. oleracea and the positive line JF2608 


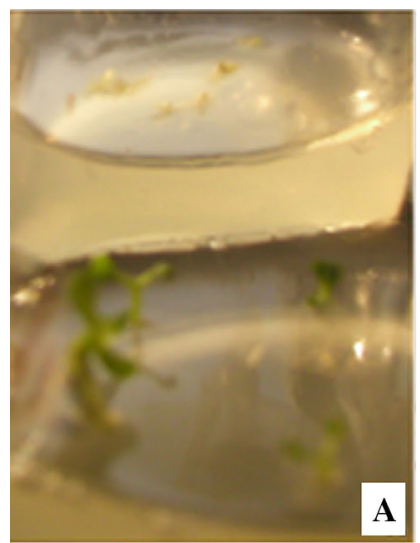

Fig. $1 F_{1}$ hybrid plant produced from intergeneric cross between $S$. alba $\times B$. oleracea-bar. a Germination of ovules from $S$. alba $\times$ B. oleracea-bar cultured on MS media. b Seedling of $S$. alba $\times$ B. oleracea-bar cultured on MS media
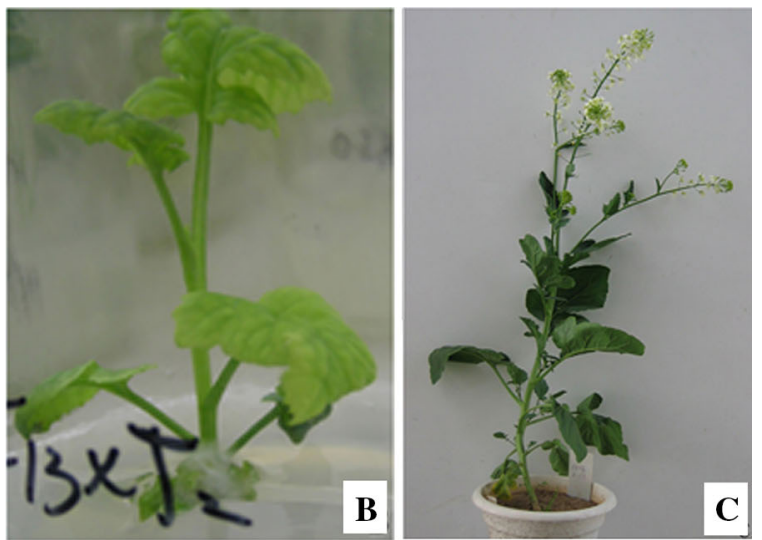

Table 1 Frequencies of intergeneric hybrid between $S$. alba and B. oleracea -bar by sexual crossing and embryo rescue

\begin{tabular}{lllll}
\hline Crosses & No. post inseminated ovaries & No. embryos obtained & No. seedlings & Percentage $(\%)^{\mathrm{b}}$ \\
\hline S. Alba $\times$ B. oleracea-bar & 410 & 17 & 13 & 3.17 \\
B. oleracea-bar $\times$ S. alba & 152 & 6 & 2 & 1.32 \\
\hline
\end{tabular}

${ }^{\mathrm{a}} B$. oleracea-bar represents transgenic lines with bar gene

${ }^{\mathrm{b}}$ Frequencies of embryo obtained equal to the number of embryo obtained/number of ovary cultured $\times 100 \%$, while hybrid production rate is equivalent to hybrid plantlets/number of ovary cultured $\times 100 \%$

Table 2 Chromosome numbers and pollen stainability of $\mathrm{F}_{1}$ intergeneric hybrid plants from S. alba $\times$ B. oleracea-bar

\begin{tabular}{lllll}
\hline Plant code & No. chromosomes & No. samples & No. pollens & \% Pollen stainability (mean \pm SD) \\
\hline JF2601 & 30 & 2 & 655 & $23.65 \pm 10.89$ \\
JF2602 & 21 & 3 & - & Sterile \\
JF2603 & 21 & 2 & - & Sterile \\
JF2604 & 21 & 3 & - & Sterile \\
JF2605 & ND & 2 & 744 & $3.57 \pm 2.27$ \\
JF2606 & 21 & 1 & - & Sterile \\
JF2607 & 21 & 3 & - & Sterile \\
JF2608 & 30 & 4 & 783 & $40.26 \pm 11.22$ \\
JF2609 & 21 & 2 & - & Sterile \\
JF2610 & 21 & 2 & 777 & $9.36 \pm 3.60$ \\
JF2611 & 21 & 3 & - & Sterile \\
B. oleracea-bar & 18 & 3 & 969 & $98.87 \pm 15.49$ \\
S. alba & 24 & 3 & 864 & $91.35 \pm 11.26$ \\
\hline
\end{tabular}

${ }^{a}$ Not determined. The exact number of chromosomes was unknown due to poor GISH result 


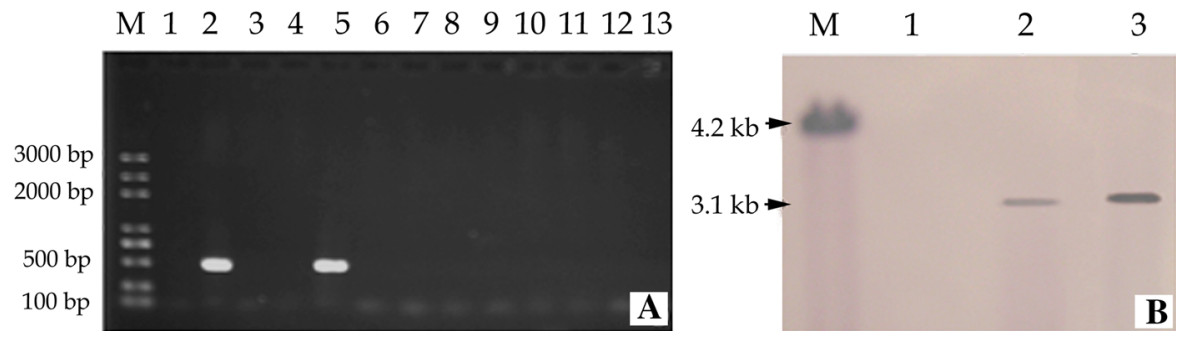

Fig. 2 Molecular characterization of the intergeneric $F_{1}$ hybrids. a PCR assay of bar gene in $\mathrm{F}_{1}$ hybrids of $S$. alba $\times B$. oleracea-bar; $M$ marker, lane 1 negative control, lane 2 positive control, lane 3-13 $\mathrm{F}_{1}$ plants of $S$. alba $\times$ B. oleracea-bar; among these, lane 5 indicates JF2608. b Southern blot assay of $\mathrm{F}_{1}$ hybrid plants; a fragment of bar gene, generated by PCR

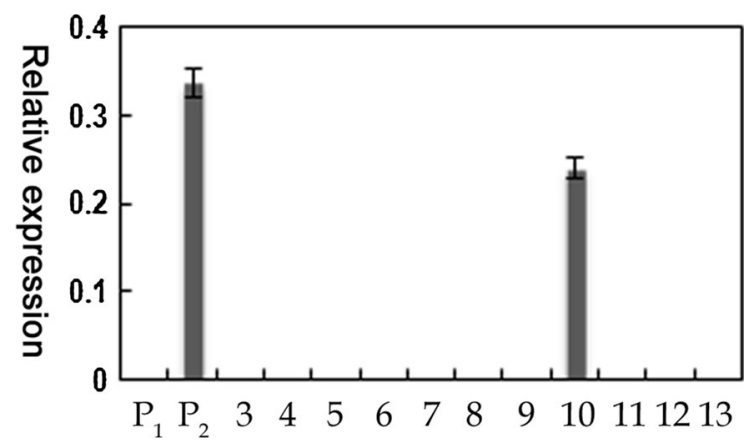

Fig. 3 qRT-PCR analysis of bar gene in $\mathrm{F}_{1}$ hybrid lines. $\mathrm{P}_{1} S$. alba, $\mathrm{P}_{2}$ B. oleracea-bar, lanes 3-13 indicate hybrid lines JF2601 to JF2611, respectively (see also in Table 2)

(Fig. 3). Finally, when all hybrid lines were sprayed with herbicide (13.5\% Basta at a 1:200 dilution), only JF2608 showed a resistance to herbicide, which was consistent with the PCR test and Southern blot analysis. Thus, the combination of results showed that the bar gene from transgenic $B$. oleracea was successfully integrated into the genome of the intergeneric hybrid; why the frequency was so low (given homozygosity of the donor) is not clear at the moment.

\section{Cytological observations}

Chromosome counting was performed using flower buds on $11 \mathrm{~F}_{1}$ hybrid plants that could grow until flowering stage (Table 2; Fig. 4). As expected, $8 \mathrm{~F}_{1}$ plants (JF2602-2604, JF2606-2607, and JF2609-2611) had 21 chromosomes, with 12 chromosomes from the $\mathrm{S}$ genome and 9 from the $\mathrm{C}$ genome (Fig. 4j). These plants were possibly the result of a failure of chromosome doubling induced by amplification of pCAMBIA3300, was used as the probe $(0.5 \mathrm{~kb}) ; M$ plasmid pCAMBIA3300 as positive control, lane 1 negative control (non-transgenic $B$. oleracea cv. 'Zhonghua Jielan'), lane 2 the genomic DNA of hybrid JF2608, lane 3 genomic DNA of transgenic B. oleracea

colchicine because they possessed the exact number of chromosomes equal to the sum of the two parental species. Intriguingly, two $\mathrm{F}_{1}$ hybrid plants (JF2601 and JF2608) showed 30 chromosomes, with 12 chromosomes from the $\mathrm{S}$ genome and 18 from the $\mathrm{C}$ genome (Fig. 4e). This result was unexpected and indicated that these plants had the entire sets of parental CC genomes, which might be caused by the generation of unreduced male gametes. In addition, one plant (JF2605) was failed to determine the chromosome constitution due to poor GISH result.

\section{Pollen viability}

Pollen fertility was also examined in the $F_{1}$ intergeneric hybrid plants. The pollen fertility of the $11 \mathrm{~F}_{1}$ hybrid plants varied greatly, ranging from $0 \%$ (completely male sterile) to $40 \%$ (partially fertile (Table 2 ). Compared with the pollen fertility of the male parent B. oleracea (99\%), most of the $F_{1}$ hybrid plants showing 21 chromosomes had a completely abortive phenotype (Table 2; Fig. 4h). However, notably, the bar gene positive line (JF2608, with 30 chromosomes) had higher pollen fertility than that of any other hybrid plants (Table 2; Fig. 4c).

Then, the semi-fertile plants (i.e. JF2601, 2605, 2608, and 2610) were further self-pollinated to generate $\mathrm{F}_{2}$ seeds. A total number of $0,2,26$ and 5 seeds respectively, were obtained from the above four $F_{1}$ plants, although some of them were poorly developed. The extremely low seed-setting rates indicated that there may exist self-incompatibility or pre-fertilization barriers. 

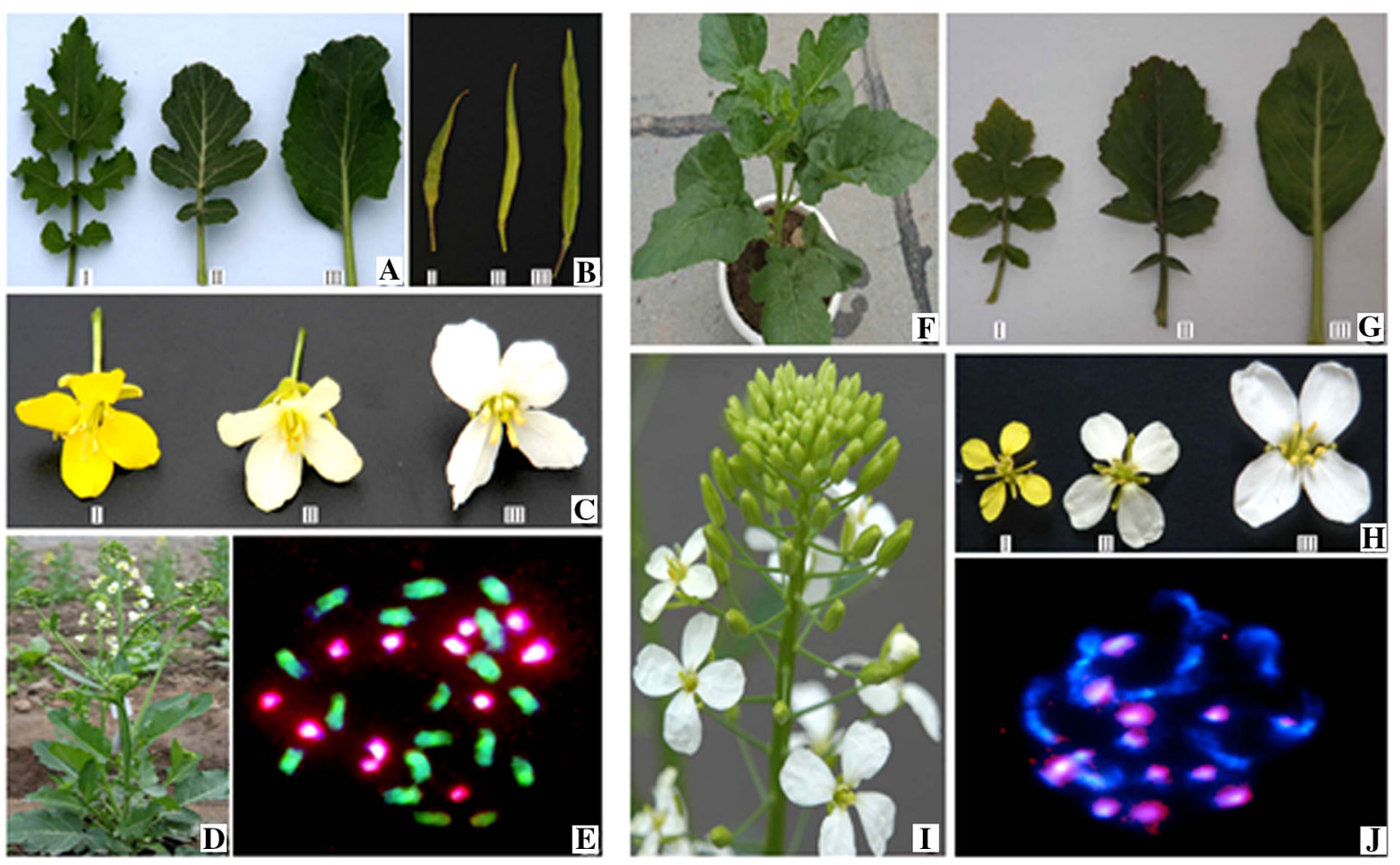

Fig. 4 Morphological and cytogenetic characterizations of hybrid plants. a-e Morphology of $S$. alba $\times$ B. oleracea-bar $\mathrm{F}_{1}$ hybrid with genome $\mathrm{CCS}=30 ;$ I S. alba, II Hybrid, III B. oleracea-bar. a Leaves, b Siliques, $\mathbf{c}$ Flowers, d Whole plants at flowering stage, e Identification of mitotic spread of semi-fertile $\mathrm{F}_{1}$ plants with 30 chromosomes consisting of $18 \mathrm{C}$ chromosomes from B. oleracea-bar (green) and $12 \mathrm{~S}$ chromosomes from $S$.

Morphology of $F_{1}$ hybrids

All $\mathrm{F}_{1}$ hybrid plants had considerable morphological features intermediate between the two parents during the process of development, which indicated that the phenotypic characters from the parents were inherited (Fig. 4).

The leaf of the hybrid plants was intermediate between those of the parents. The lower part of the hybrid leaf was similar to that of $S$. alba, with deeply pinnate lobes. However, the upper portion of the leaf was lanceolate and more similar to the leaf shape of $B$. oleracea. Additionally, trichomes were present on the leaf petioles and stem, which are not commonly found in B. oleracea (Fig. 4a, g).

The flowers also showed patterns similar to those of the parents. The flower buds of $F_{1}$ hybrid plants were numerous and widely spaced on the raceme, similar to those of $S$. alba but smaller than those of B. oleracea. alba (red or pale-red) by genomic in situ hybridization (GISH); f-j Morphology of S.alba $\times$ B.oleracea-bar $\mathrm{F}_{1}$ hybrid with $\mathrm{CS}=21$. I S. alba, II Hybrid, III B. oleracea. f Seedling, $\mathbf{g}$ leaves, $\mathbf{h}$ flowers, $\mathbf{i}$ buds and flowers, $\mathbf{j}$ GISH for the sterile $\mathrm{F}_{1}$ plants with 21 chromosomes consisting of $9 \mathrm{C}$ chromosomes (Blue) and $12 \mathrm{~S}$ chromosomes (red)

Compared with the flowers of the female parent $S$. $a l b a$, the flowers of $F_{1}$ hybrids were different colors, mostly white or milky (Fig. 4c, d, h, i). Moreover, notably, compared with the flowering time of either parent, the flowering time of $F_{1}$ hybrids was slightly earlier and longer than those of the parents.

The silique of $F_{1}$ hybrid plants was intermediate in length (Fig. 4b). Additionally, mature hybrid plants had more branches than either parent. Of note, although the genome sets were different, characters such as the shape of the leaf, stem, flower and silique of the $\mathrm{F}_{1}$ hybrid plants with 21 chromosomes resembled those of the plants with 30 chromosomes (Fig. 4). The combination of the above observations indicated that the hybrid plants inherited characters of both parents effectively. 
Agronomic trait performance

The $\mathrm{F}_{2}$ seeds of JF2605, JF2608, and JF2610 were first sown in pots in greenhouse but only JF2608 could germinated. A total of five seedlings from JF2608 were obtained and pricked out to the field. At flowering stage, the JF2608 $\mathrm{F}_{2}$ plants were bagged to produce $\mathrm{F}_{3}$ seeds. Only two plants (JF2608-2, JF26085) could produce enough seeds $(>1.0 \mathrm{~g})$ for the analysis of oil content and seed weight. Economically important agronomic traits were recorded at mature stage.

Each hybrid silique had from 2 to 6 seeds, with a mean seed size of $2.03 \mathrm{~mm}$. The average seed size was 2.41 and $1.86 \mathrm{~mm}$ for $S$. alba and B. oleracea, respectively (Table 3 ). The results were similar for the thousand-seed weight and oil content of the $F_{2}$ plants and their parents. The hybrid had an average 1000 -seed weight of $4.27 \mathrm{~g}$, which was heavier than that of B. oleracea at $3.62 \mathrm{~g}$ but markedly lighter than that of $S$. alba at $5.59 \mathrm{~g}$ (Table 3). The seed oil content in hybrid was low (29.2\%) close to the mean of its two parents $(31 \%)$, indicating that there was no heterosis for this trait. However, hybrid vigor was observed for plant height. Compared with both parents, the hybrid plants were $19-63 \%$ taller, reaching a height of $74 \mathrm{~cm}$ (Table 3).

\section{Alternaria brassicae resistance analysis}

The disease assessment of JF2608 $\mathrm{F}_{3}$ hybrid plants and the two parents inoculated with the pathogen for 6 days was shown in Fig. 5. Among the $38 \mathrm{JF}^{2608 \mathrm{~F}_{3}}$ hybrid plants (14 derived from JF2608-2 and 24 from JF2608-5), almost half showed high resistance to $A$. brassicae (rating of 1-2), similar to that of the female parent $S$. alba. Some other lines had moderate resistance (rating of 3-4), while the remaining 4 lines

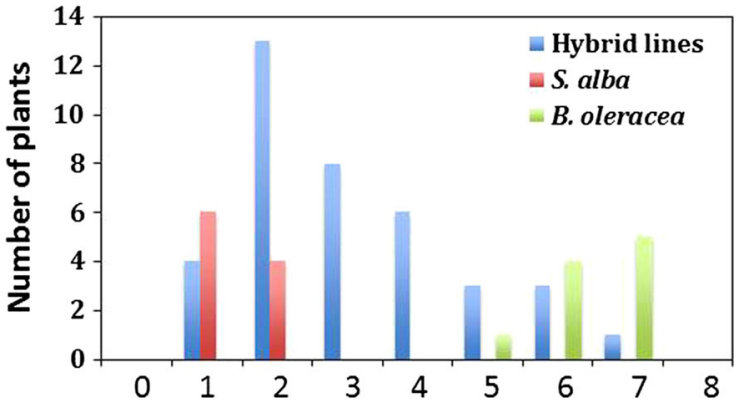

Fig. 5 Evaluation of $A$. brassicae resistance. Thirty eight $\mathrm{F}_{3}$ hybrid plants (derived from two $\mathrm{F}_{2}$ plants) and 10 plants of each parent were used to test the resistance to fungal pathogen $A$. brassicae. Scale 0 complete resistance, Scale 1-2 high resistance, Scale 3-4 moderate resistance, Scale 5-7 susceptibility; Scale 8 , complete susceptibility

showed apparent susceptibility, with symptoms similar to those of the $B$. oleracea parent. For the $\mathrm{F}_{3}$ hybrid plants, the average rating of disease severity was 3.1, whereas the rating was 1.4 and 6.4 for the female parents S. alba and B. oleracea, respectively (Fig. 5).

\section{Discussion}

By distant sexual hybridization coupling with embryo rescue, transgenic intergeneric hybrids between $S$. alba and B. oleracea-bar were obtained for the first time. The hybridity could be confirmed by both cytological investigation and morphological observation. Out of 410 pollinations, $13 \mathrm{~F}_{1}$ hybrid seedlings were obtained from the direct cross ' $S$. alba $\times B$. oleracea', representing $\sim 3 \%$ of the entire number of rescued ovaries. The frequency of the reciprocal crosses was much lower (Table 1), indicating that true hybrids would be more easily obtained with $S$. alba as the maternal parent. Our observation was consistent with the results from Wei et al. (2007) who

Table 3 Comparisons of agronomic traits in intergeneric hybrid $F_{2}$ plants and their parental lines

\begin{tabular}{lllll}
\hline & $\begin{array}{l}\text { Seed size } \\
(\mathrm{mm})\end{array}$ & $\begin{array}{l}1000 \text { seed weight } \\
(\mathrm{g})\end{array}$ & $\begin{array}{l}\text { Oil content } \\
(\%)\end{array}$ & $\begin{array}{l}\text { Mature plant height }(\mathrm{cm}) \\
(\mathrm{mean} \pm \mathrm{SD})\end{array}$ \\
\hline S. alba & 2.41 & 5.59 & 25.3 & $62.1 \pm 9.70$ \\
$\begin{array}{l}\text { B. oleracea-bar } \\
\text { S. alba } \times \text { B. oleracea-bar }(\mathrm{JF} 2608\end{array}$ & 1.86 & 3.62 & 36.6 & $45.4 \pm 7.60$ \\
$\left.\mathrm{~F}_{2}\right)$ & 2.03 & 4.27 & 29.2 & $74.2 \pm 11.84$ \\
\hline
\end{tabular}


used non-transgenic B. oleracea and S. alba as plant materials. A pre-fertilization barrier might be the primary explanation for the lower success rate, as demonstrated by the limited growth of $B$. carinata pollen tubes in B. oleracea (Dey et al. 2015). However, our finding was contradict to those of Momotaz et al. (1998) who concluded that hybrids could be obtained from the combination of $B$. oleracea var. alboglabra $\times S$. alba but not vice versa. This contradiction could be either due to different genotypes they used, or limited number of buds (only $33 \sim 45)$ they had pollinated. Generally, hybridizations are more successful when the maternal parent has a higher chromosome number (Justin and Schemske 1998; Cheung et al. 2015).

Theoretically, the somatic number of chromosomes of intergeneric sexual $\mathrm{F}_{1}$ hybrid between $S$. alba and $B$. oleracea should be 21 , or 42 if doubled. Indeed, our cytological investigations indicated that most of the $\mathrm{F}_{1}$ hybrid plants had the expected 21 chromosomes (one set each of C and S chromosomes, Table 2), confirming the same observation by Wei et al. (2007) in nonetransgenic $S$. alba $\times$ B. oleracea. However, we also obtained a few unexpected plants with 30 chromosomes, consisting of one $\mathrm{S}$ chromosome set and two $\mathrm{C}$ chromosome sets. These findings were consistent with those of previous research (Inomata 2002; Wei et al. 2007). Based on these results, we deduced that these plants were generated by unreduced male gametes from parental B. oleracea. Although the occurrence of unreduced male or female gametes is extremely low, this phenomenon has been reported in the family Brassicaceae (Ayotte et al. 1988; Fominaya et al. 2005). An alternative possibility is that the chromosome set was partially doubled by colchicine treatment (namely only $\mathrm{C}$ chromosome set was doubled).

We also observed the association between pollen viability and chromosome constitution of the intergeneric hybrid. Almost all $\mathrm{F}_{1}$ plants with 21 chromosomes were highly male sterile, while those plants with more chromosomes tended to have higher level of pollen viability (Table 2). Male fertility could be further improved by backcrossing with $B$. oleracea (Wei et al. 2007), although Hansen and Earle (1997) argued that chromosome doubling should not help.

The generation of disease (A. brassicae) and herbicide (Bastar) tolerant intergeneric hybrids were of great interest, since they could be used as valuable intermediate materials to transfer genes from $S$. alba to
B. oleracia or B. napus via resynthesis of B. napus with B. oleracea-bar and B. rapa. The existence of bar gene will greatly facilitate the identification of hybridity and transfer of valuable traits into other genotypes. Basically, all $F_{1}$ offspring should carry a bar gene and thus was transgenic positive, if a homozygous $B$. oleracia-bar was used as parent. However in this study, based on both the PCR assay and Southern blot analysis, only one out of 11 hybrid $\mathrm{F}_{1}$ was showed to be transgenic positive; therefore, the efficiency of inheritance of bar gene from parent to $F_{1}$ hybrids $(\sim 10 \%)$ was much lower than expected. In addition, our previous work showed that almost half the total offspring were transgenic positive in the hybridization of $B$. oleracea-bar $\times B$. rapa $(\mathrm{Li}$ et al. 2006). The low proportion of transgenic plants in the hybrids described here is surprising. Low transmission rate of bar to subseqent might be partially a consequence of genome incompatibility between the parents $S$. alba and B. oleracea (Hansen and Earle 1997; Halfhill et al. 2002) or perhaps some gene silencing mechanism operating in an intergeneric hybrids.

Authors' contribution XF and GL conceived and designed the study. JL, CZ, CG, JL, LL, RL, and WW participated in the experiments. LJ and GL analyzed the data. JL and GL bred the material. All authors drafted the manuscript and approved the final manuscript.

Funding This work was financially supported by the National Natural Science Foundation of China (31371663, 31771735, 31101124, and 31401720), Special Fund for Agro-scientific Research in the Public Interest (201203096), and Modern Agricultural Technology System (CARS-0013).

\section{Compliance with ethical standards}

Conflict of interest The authors declare that the research was conducted in the absence of any commercial or financial relationships that could be construed as a potential conflict of interest.

Open Access This article is distributed under the terms of the Creative Commons Attribution 4.0 International License (http:// creativecommons.org/licenses/by/4.0/), which permits unrestricted use, distribution, and reproduction in any medium, provided you give appropriate credit to the original author(s) and the source, provide a link to the Creative Commons license, and indicate if changes were made. 


\section{References}

Ayotte R, Harney PM, Machado VS (1988) The transfer of triazine resistance from Brassica napus L to Brassica oleracea L. 2. morphology, fertility and cytology of the $\mathrm{F}_{1}$ hybrid. Euphytica 37:189-197

Bodnaryk RP, Lamb RJ (1991) Mechanisms of resistance to the flea beetle, Phyllotreta cruciferae (Goeze), in mustard seedlings, Sinapis alba L. Can J Plant Sci 71:13-20

Brown J, Brown A, Davis J, Erickson D (1997) Intergeneric hybridization between Sinapis alba and Brassica napus. Euphytica 93:163-168

Chen LP, Zhang MF, Li CS, Hirata Y (2005) Production of interspecific somatic hybrids between tuber mustard (Brassica juncea) and red cabbage (Brassica oleracea). Plant Cell Tissue Org Cult 80:305-311

Cheung KW, Razeq FM, Sauder CA, James T, Martin SL (2015) Bidirectional but asymmetrical sexual hybridization between Brassica carinata and Sinapis arvensis (Brassicaceae). J Plant Res 128:469-480

Chevre AM, Eber F, Margale E, Kerlan MC, Primard C, Vedel F, Delseny M, Pelletier G (1994) Comparison of somatic and sexual Brassica napus - Sinapis alba hybrids and their progeny by cytogenetic studies and molecular characterization. Genome 37:367-374

Dey SS, Sharma K, Dey RB, Kumar GMS, Singh D, Kumar R, Parkash C (2015) Inter specific hybridization (Brassica carinata $\times$ Brassica oleracea) for introgression of black rot resistance genes into Indian cauliflower $(B$. oleracea var. botrytis L.). Euphytica 204:149-162

Fominaya A, Linares C, Loarce Y, Ferrer E (2005) Microdissection and microcloning of plant chromosomes. Cytogenet Genome Res 109:8-14

Gong ZH, He YK, Wang M (1994) Studies on the resistance of intergeneric hybrids cabbage $\times$ white mustard to Alternaria leaf spot. Acta Hortic Sin 21:401-403

Gribova TN, Kamionskaia AM, Skriabin KG (2006) Optimization of the protocol for constructing transgenic plants of the white cabbage Brassica oleracea var. capitata. Prikl Biokhim Mikrobiol 42:593-598

Halfhill MD, Millwood RJ, Raymer PL, Stewart CN Jr (2002) Bt-transgenic oilseed rape hybridization with its weedy relative, Brassica rapa. Environ Biosaf Res 1:19-28

Hansen LN, Earle ED (1997) Somatic hybrids between Brassica olerace $\mathrm{L}$ and Sinapis alba $\mathrm{L}$ with resistance to Alternaria brassicae (Berk) Sacc. Theor Appl Genet 94:1078-1085

Inomata N (2002) A cytogenetic study of the progenies of hybrids between Brassica napus and Brassica oleracea, Brassica bourgeaui, Brassica cretica and Brassica montana. Plant Breed 121:174-176

Justin R, Schemske DW (1998) Pathways, mechanisms, and rates of polyploid formation in flowering plants. Annu Rev Ecol Syst 29:467-501
Kaneko Y, Bang SW (2014) Interspecific and intergeneric hybridization and chromosomal engineering of Brassicaceae crops. Breed Sci 64:14-22

Lelivelt CL, Leunissen EH, Frederiks HJ, Helsper JP, Krens FA (1993) Transfer of resistance to the beet cyst nematode (Heterodera Schachtii Schm.) from Sinapis alba L. (white mustard) to the Brassica napus L. gene pool by means of sexual and somatic hybridization. Theor Appl Genet 85:688-696

Li J, Fang X, Wang Z, Li J, Luo L, Hu Q (2006) Transgene directionally integrated into C-genome of Brassica napus. Chin Sci Bull 51:1578-1585

Momotaz A, Kato M, Kakihara F (1998) Production of intergeneric hybrids between Brassica and Sinapis species by means of embryo rescue techniques. Euphytica 103:123-130

Nothnagel T, Budahn H, Straka P, Schrader O (1997) Successful backcrosses of somatic hybrids between Sinapis alba and Brassica oleracea with the Brassica oleracea parent. Plant Breed 116:89-97

Nothnagel T, Budahn H, Schrader O, Klocke E (2013) Intergeneric hybridization between Brassica oleracea and Eruca sativa. Acta Hort 1005:97-103

Piętka T, Krzymański J, Krótka K, Bartkowiak-Broda I (2014) Double low white mustard (Sinapis alba L. syn. Brassica hirta) is a source of protein and oil. Rośliny Oleiste 35:21-35

Scholze P, Kramer R, Ryschka U, Klocke E, Schumann G (2010) Somatic hybrids of vegetable Brassicas as source for new resistances to fungal and virus diseases. Euphytica 176:1-14

Wang YP, Zhao XX, Sonntag K, Wehling P, Snowdon RJ (2005) Behaviour of Sinapis alba chromosomes in a Brassica napus background revealed by genomic in situ hybridization. Chromosome Res 13:819-826

Wang GX, Tang Y, Yan H, Sheng XG, Hao WW, Zhang L, Lu K, Liu F (2011) Production and characterization of interspecific somatic hybrids between Brassica oleracea var. botrytis and B. nigra and their progenies for the selection of advanced pre-breeding materials. Plant Cell Rep 30:1811-1821

Wei WH, Zhang SF, Li J, Wang LJ, Chen B, Wang Z, Luo LX, Fang XP (2007) Cytogenetic analysis of $F_{1}, F_{2}$ and $B_{1}$ plants from intergeneric sexual hybridization between $\mathrm{Si}$ napis alba and Brassica oleracea by genomic in situ hybridization. Plant Breed 126:392-398

Wei F, Gao GZ, Wang XF, Dong XY, Li PP, Hua W, Wang X, Wu XM, Chen H (2008) Quantitative determination of oil content in small quantity of oilseed rape by ultrasoundassisted extraction combined with gas chromatography. Ultrason Sonochem 15:938-942

Wu G, Zhang L, Wu Y, Cao Y, Lu C (2010) Comparison of five endogenous reference genes for specific PCR detection and quantification of Brassica napus. J Agric Food Chem $58: 2812-2817$ 